\title{
Intrusive memories to traumatic footage: the neural basis of their encoding and involuntary recall
}

\author{
I. A. Clark ${ }^{1}$ t, E. A. Holmes ${ }^{2,3}$, M. W. Woolrich ${ }^{4}$ and C. E. Mackay ${ }^{1 *}$ \\ ${ }^{1}$ Department of Psychiatry, University of Oxford, Warneford Hospital, Oxford OX3 7NG, UK \\ ${ }^{2}$ Medical Research Council Cognition and Brain Sciences Unit, 15 Chaucer Road, Cambridge CB2 7EF, UK \\ ${ }^{3}$ Division of Psychology, Department of Clinical Neuroscience, Karolinska Institutet, Stockholm, Sweden \\ ${ }^{4}$ Department of Psychiatry, Oxford Centre for Human Brain Activity (OHBA), Warneford Hospital, Oxford OX3 7NG, UK
}

Background. A hallmark symptom after psychological trauma is the presence of intrusive memories. It is unclear why only some moments of trauma become intrusive, and how these memories involuntarily return to mind. Understanding the neural mechanisms involved in the encoding and involuntary recall of intrusive memories may elucidate these questions.

Method. Participants $(n=35)$ underwent functional magnetic resonance imaging (fMRI) while being exposed to traumatic film footage. After film viewing, participants indicated within the scanner, while undergoing fMRI, if they experienced an intrusive memory of the film. Further intrusive memories in daily life were recorded for 7 days. After 7 days, participants completed a recognition memory test. Intrusive memory encoding was captured by comparing activity at the time of viewing 'Intrusive scenes' (scenes recalled involuntarily), 'Control scenes' (scenes never recalled involuntarily) and 'Potential scenes' (scenes recalled involuntarily by others but not that individual). Signal change associated with intrusive memory involuntary recall was modelled using finite impulse response basis functions.

Results. We found a widespread pattern of increased activation for Intrusive $v$. both Potential and Control scenes at encoding. The left inferior frontal gyrus and middle temporal gyrus showed increased activity in Intrusive scenes compared with Potential scenes, but not in Intrusive scenes compared with Control scenes. This pattern of activation persisted when taking recognition memory performance into account. Intrusive memory involuntary recall was characterized by activity in frontal regions, notably the left inferior frontal gyrus.

Conclusions. The left inferior frontal gyrus may be implicated in both the encoding and involuntary recall of intrusive memories.

Received 13 November 2014; Revised 19 June 2015; Accepted 11 September 2015

Key words: Dorsolateral prefrontal cortex, flashbacks, functional magnetic resonance imaging, intrusive memories, left inferior frontal gyrus.

\section{Introduction}

The majority of people will experience or witness a traumatic event during their lifetime and a significant minority will develop post-traumatic stress disorder (PTSD) (Breslau et al. 1998; American Psychiatric Association, 2013). A hallmark symptom of PTSD is the occurrence of intrusive memories - involuntary images of the trauma intruding into consciousness (Brewin, 2013). We lack understanding of why only some moments within a trauma are (re)experienced

\footnotetext{
* Address for correspondence: C. E. Mackay, Department of Psychiatry, University of Oxford, Warneford Hospital, Warneford Lane, Oxford, OX3 7NG.

(Email: clare.mackay@psych.ox.ac.uk)

† Present address: Wellcome Trust Centre for Neuroimaging, Institute of Neurology, University College London, 12 Queen Square, London WC1N 3BG, UK.
}

as intrusive memories and how these moments involuntarily return to mind. Processing at the time of trauma (peritraumatic processing) - i.e. during memory encoding - has been implicated in both later PTSD and intrusive memory development (Ehlers \& Clark, 2000; Ozer et al. 2003; American Psychiatric Association, 2013; Brewin, 2014). Investigating the neural mechanisms during encoding may add to our understanding of intrusive memories. The current study investigated a hypothesized neural 'signature' during the encoding of an experimental analogue of trauma (Bourne et al. 2013), and the involvement of this signature in later intrusive memory involuntary recall.

Due to the nature of PTSD, the wealth of neuroimaging work has been conducted in PTSD patients with established symptoms (Lanius et al. 2006; Hughes \& Shin, 2011). Such research has often used symptom provocation paradigms, which involve exposing

This is an Open Access article, distributed under the terms of the Creative Commons Attribution licence (http://creative commons.org/licenses/by/4.0/), which permits unrestricted re-use, distribution, and reproduction in any medium, provided the original work is properly cited. 
PTSD patients to reminders of their trauma while undergoing neuroimaging (Rauch et al. 1996; Shin et al. 1997, 2004, 2006; Lanius et al. 2006; Hughes \& Shin, 2011). Neurocircuitry models from this work suggest that PTSD is characterized by increased amygdala (and other limbic) activation and reduced ventromedial prefrontal cortex activation (Rauch et al. 1998, 2006). A recent model by Admon et al. (2013) suggested that abnormalities in the amygdala and dorsal anterior cingulate cortex are predisposing, while abnormal interactions between the hippocampus and ventromedial prefrontal cortex arise after developing PTSD (Admon et al. 2013). However, patient studies can tell us little about how intrusive memories are formed since they cannot examine the original encoding of the trauma.

Studying the neural correlates of real-life trauma is unfeasible, but intrusive memories can be experimentally induced using an experimental analogue - the trauma film paradigm (Lazarus, 1964; Holmes \& Bourne, 2008). Participants view footage of real-life scenes of death and serious injury, in line with the fifth edition of the Diagnostic and Statistical Manual of Mental Disorders (DSM-5; American Psychiatric Association, 2013) definition for psychological trauma. Combining the trauma film with neuroimaging allows a prospective design to study intrusive memory encoding and involuntary recall.

We recently conducted, to our knowledge, the only study to date investigating the neural basis of intrusive memory encoding (Bourne et al. 2013). Results suggested a widespread neural signature at the time of viewing scenes that later became intrusive memories, including increases in activation in the amygdala, striatum, rostral anterior cingulate cortex, thalamus and ventral occipital cortex.

In particular, two regions (and only these) seemed to distinguish between scenes that became intrusive memories for an individual and scenes that had the 'potential' to become intrusive memories (i.e. scenes of emotional content recalled involuntarily by some participants, but not that individual); the left inferior frontal gyrus (IFG) and middle temporal gyrus (MTG) (Bourne et al. 2013). These results at encoding partially mirror the 'subsequent memory effect' found in non-traumatic memory (Paller \& Wagner, 2002; Kensinger \& Corkin, 2004). The subsequent memory effect suggests predictive differences at encoding for items that are later deliberately recalled relative to items that are not recalled in left prefrontal regions and bilateral middle temporal regions areas that include the left IFG and MTG. We therefore sought to ask whether these two regions would also predict moments of the film that would be recalled involuntarily. Another possible explanation for our previous results is that intrusive memory scenes were simply better recognized than potential scenes. These encoding results require replication, and additionally for recognition memory to be taken into account.

Our second question concerns the neural basis of intrusive memory involuntary recall. To our knowledge, no study has captured the neural activation at the moment of intrusive memory involuntary recall - that is, the moment when a participant experiences an intrusive memory while undergoing functional magnetic resonance imaging (fMRI). Symptom provocation studies indicate increased activity in limbic and paralimbic areas, suggesting that these regions may be involved in intrusive memory involuntary recall (Rauch et al. 1996; Liberzon et al. 1997; Shin et al. 1999; Osuch et al. 2001). However, while patients may (or may not) experience intrusive memories during scanning, the neural mechanisms of their involuntary recall remain unknown as an intrusive memory could have occurred at any point during symptom provocation. Further, other symptoms with different underpinning may be implicated during symptom provocation (Bryant et al. 2013; Pietrzak et al. 2013). In a separate vein, one study using healthy participants has shown that the involuntary recall of picture stimuli, compared with their voluntary recall, has been associated with the middle and superior frontal gyri (Hall et al. 2008). Whether these results can be extrapolated to intrusive memories of traumatic stimuli is unknown.

We note that part of the data acquired and presented here (the fMRI data concerning encoding) is also used elsewhere (Clark et al. 2014) in combination with our previous work (Bourne et al. 2013). Using different analysis techniques we attempted to investigate a second separate question - one of prediction instead of association. That is, could we 'learn' the brain activity associated with later intrusive memories in order to predict, from new unseen brain activity, 'future' intrusive memories? On the other hand, here, we report the differences in brain activity during scenes that were later recalled involuntarily by that participant (intrusive scenes), compared with scenes recalled involuntarily by previous participants, but not that individual (potential scenes). The work presented in the present paper therefore attempts to identify regions that may differentiate between intrusive and potential scenes. Our parallel work (Clark et al. 2014) attempts to quantify the extent that solely the peritraumatic brain activity can predict intrusive memories. Thus, although we recognize that we use the same component of the dataset in two different papers, we use it to address two distinctly different questions.

The current experiment investigated the encoding and involuntary recall of intrusive memories of experimental trauma. We first sought to replicate our previous findings of widespread increases in neural activation at the time of viewing scenes that caused 
intrusive memories relative to scenes that did not. Specifically, we predicted that activation in the left IFG and MTG would distinguish intrusive memory scenes from 'Potential' scenes (scenes of emotional content recalled involuntarily by previous participants, but not that individual). Further, to take into account possible signal changes due to better recognition memory for intrusive compared with potential scenes, we reconfigured the fMRI time series into film 'stills' to repeat our encoding analysis using only correctly recognized film picture stills. Finally, we sought to investigate the neural mechanisms of intrusive memory involuntary recall, modelling brain activity while participants experienced an intrusive memory during fMRI. To adaptively capture the moment of involuntary recall we modelled the fMRI time series data using finite impulse response basis functions.

\section{Method}

\section{Participants}

A total of 41 participants were recruited from the local community. Data could not be analysed for six participants (online Supplementary material). This left 35 participants (mean age $=22.43$ years, s.D. $=7.52 ; 29$ female, six male) with no reported current or previous psychiatric history. The study was approved by the University of Oxford Central University Research Ethics Committee. All participants provided written informed consent and were reimbursed $£ 25$ (US \$40).

\section{Behavioural measures}

Trauma film viewing

The experimental procedure is shown in Fig. 1. After completing baseline and mood measures (online Supplementary material) participants viewed traumatic film footage, including scenes of actual and threatened death and serious injury, while undergoing fMRI. The film comprised 15 short clips which included 20 Possible intrusive scenes and 16 Control scenes. Scene type was determined using data from approximately 200 participants who had taken part in previous behavioural experiments. 'Possible' scenes were scenes that had induced intrusive memories in previous participants (e.g. emergency personnel at an accident with an injured victim), 'Control' scenes were those that had never induced intrusive memories (e.g. emergency personnel around the accident but no visible death or injury). Possible scenes were later classified as either 'Intrusive' scenes (recalled involuntarily by that participant) or 'Potential' scenes (not recalled involuntarily by that participant, but recalled involuntarily by previous participants) depending on the diary data (see Intrusive memory diary below). All scenes had unique topic content to facilitate intrusive memory identification. Scene length was matched as closely as possible between Possible (length, 5-37 s; mean 22.5 s) and Control scenes (length, 5-36 s; mean 16.4 s) $\left(t_{34}=1.94\right.$, N.s. $)$; see the online Supplementary material (online Supplementary Tables S1 and S2) for the exact duration of each scene. Scenes were distributed evenly throughout the whole film. These constraints were included to take into account the relative slowness of the haemodynamic response (Buxton et al. 2004).

\section{Intrusive memory involuntary recall during $f M R I$}

On film completion, participants were briefly removed from the scanner to complete mood ratings. As per previous trauma film paradigm experiments (Holmes et al. 2004), participants were instructed as how to identify intrusive memories - defined as: (1) moments of the film spontaneously popping into mind unexpectedly (rather than the participant purposefully recalling the film); and (2) mental images, e.g. taking the form of pictures or sounds.

Participants then returned into the scanner. Participants were asked to lie in the scanner for $6 \mathrm{~min}$ and respond with a button press if they experienced an intrusive memory of the film (i.e. if the film spontaneously popped into their mind). To minimize experimental demands, it was made clear to the participants that they may - or may not - experience any intrusive memories during the scan. This allowed us to capture the moment of intrusive memory involuntary recall while participants were in the scanner undergoing fMRI.

\section{Intrusive memory diary}

Participants kept a daily intrusive memory diary for the following week (Holmes et al. 2004). Participants were asked to write the content of any intrusive memory (e.g. the car hitting the boy) and their emotional rating of the intrusive memory, from 1 'very negative' to 5 'very positive'. All content descriptions were checked to confirm they matched a specific film scene. Intrusive memories experienced during the intrusive memory involuntary recall scan were included in the diary. From the diary Intrusive and Potential scenes we derived from the Possible scenes retrospectively for each participant. That is, 'Intrusive' scenes were those matched with the involuntary memories reported in the diary, and 'Potential' scenes were the remaining Possible scenes that did not return as intrusive memories for that participant.

\section{Film still recognition memory test}

At 1 week post-film viewing participants performed a yes/no recognition memory test containing 201 picture 


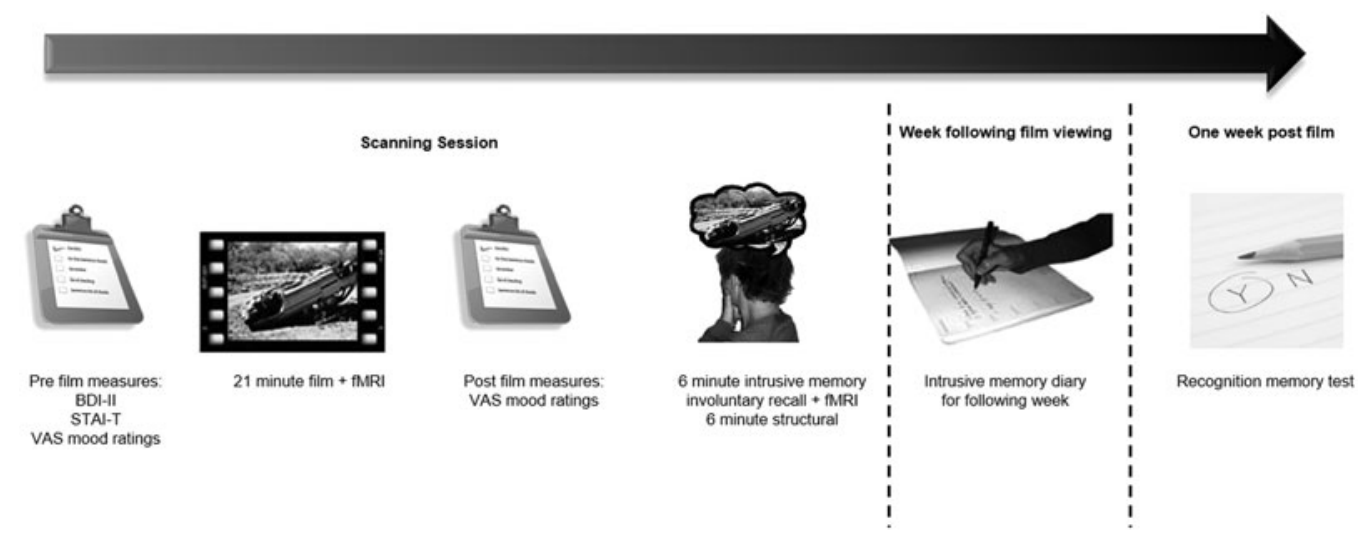

Fig. 1. Experimental procedure. Participants completed baseline questionnaires and measures of their current mood. They then viewed film footage with traumatic content, including scenes of death and serious injury, while undergoing functional magnetic resonance imaging (fMRI). On film completion participants were removed from the scanner and mood measurements were administered. Participants were then trained to identify intrusive memories. They were then returned to the scanner indicating with a button press if they experienced an intrusive memory of the film while undergoing fMRI. For the following week participants kept a diary of any further intrusive memories, returning at 1 week to perform a recognition memory test of the film contents. BDI-II, Beck Depression Inventory-II; STAI-T, State-Trait Anxiety Inventory, trait scale; VAS, visual analogue scale.

stills; 103 from the film (51 from Control scenes, 52 from Potential and Intrusive scenes) and 98 foils see the online Supplementary material.

\section{fMRI data acquisition}

All fMRI imaging data (trauma film viewing and intrusive memory involuntary recall) were acquired on a 3-T Siemens TIM Trio System with a 12-channel head coil (voxel resolution $=3 \times 3 \times 3 \mathrm{~mm}^{3}$; repetition time $=$ $3 \mathrm{~s}$; echo time $=30 \mathrm{~ms}$ ). T1-weighted structural images were acquired for subject registration using a magnetization prepared rapid gradient echo (MPRAGE) sequence (voxel resolution $=1 \times 1 \times 1 \mathrm{~mm}^{3}$; repetition time $=2040 \mathrm{~ms}$; echo time $=4.7 \mathrm{~ms}$ ).

\section{fMRI data analysis}

Analyses were performed using FEAT (fMRI Expert Analysis Tool) version 6.0 (http://www.fmrib.ox.ac. $\mathrm{uk} / \mathrm{fsl}$ ). Data were pre-processed using FEAT's default options: motion correction applied using MCFLIRT and fieldmaps with an echo planar imaging (EPI) spacing of $0.49 \mathrm{~ms}$ and echo time of $22 \mathrm{~ms}$; Gaussian spatial smoothing applied with a full width half maximum of $5 \mathrm{~mm}$; brain matter separated from nonbrain using a mesh deformation approach; high-pass temporal filtering applied with a cut-off of $100 \mathrm{~s}$.

\section{Intrusive memory encoding}

Analysis was performed at a whole-brain level. The three event types (Intrusive, Potential, Control) were specified for each participant in the general linear model with a fourth variable of no interest to model text slides (which provided information concerning each film clip). The model was applied voxel-wise to the pre-processed imaging data. First-level withinsubject analysis was performed using FILM (FMRIB's Improved Linear Model). Voxel-wise group analysis was performed in Montreal Neurological Institute (MNI) 152 standard space using FLAME (FMRIB's Local Analysis of Mixed Effects) stage 1 (Beckmann et al. 2003; Woolrich et al. 2004; Woolrich, 2008). $z$-Statistic images were thresholded at $z>2.3$ and a family-wise error corrected cluster significance threshold of $p<0.05$ (Forman et al. 1995).

Following whole-brain analysis, percentage blood oxygen level-dependent (BOLD) signal change was extracted from the left IFG and MTG using predefined regions of interest (ROIs). The left IFG and MTG were defined as regions that were significantly activated in the Bourne et al. (2013) results on a whole-brain basis in the Intrusive (referred to as Flashback) $v$. Potential contrast, but not for the Intrusive $v$. Control contrast.

To control for any effect of Intrusive scenes being better recognized than Potential scenes we performed an additional analysis (as above) using only Intrusive recognized picture stills and Potential recognized picture stills (identified by the recognition memory test) with each still modelled for $0.5 \mathrm{~s}$.

\section{Intrusive memory involuntary recall}

We recruited nine additional participants to act as a control group (online Supplementary material). Participants underwent a 6 min scan randomly pressing a button approximately 5-10 times. 
Analysis was performed at the whole-brain level. For both groups (intrusive memory involuntary recall and control button press) 3-s wide (the repetition time) finite impulse response (FIR) basis functions modelled consecutive 'time bins' surrounding the button press (Diederen et al. 2010). To take into account the approximate $6 \mathrm{~s}$ delay in haemodynamic response, the time bins were placed from -3 to $+12 \mathrm{~s}$ in relation to the button press - resulting in five time bins for each button press. The five time bins were entered into a single general linear model and applied to the pre-processed data in FILM for each participant. The FIR basis function was modelled as a single basis function with a $0 \mathrm{~s}$ phase shift and $3 \mathrm{~s}$ time window. Exploratory groupwise analysis was performed at the whole-brain level in MNI standard space using FLAME with $z$ statistic images thresholded at $z>1.7$ and a family-wise errorcorrected cluster significance threshold of $p<0.05$.

Following whole-brain analysis, percentage BOLD signal change was extracted from ROIs showing significant activation in the intrusive memory involuntary recall $v$. control button press contrast and the reverse contrast in any of the time bins. Additional signal change was extracted from the precentral gyrus to compare motor activity. The precentral gyrus was created from the Oxford-Harvard cortical and subcortical probabilistic anatomical atlas thresholded at a minimum probability of $20 \%$.

\section{Ethical standards}

All procedures contributing to this work comply with the ethical standards of the relevant national and institutional committees on human experimentation and with the Helsinki Declaration of 1975, as revised in 2008.

\section{Results}

\section{Behavioural results}

Baseline measures and mood change are reported in the online Supplementary material.

In terms of the main outcome of interest, participants reported a total of 303 intrusive memories that could be matched in content to the film from the 1-week diary and in the scanner soon after film viewing (mean per participant $=8.66$, S.D. $=7.15$ ). A further 13 intrusive memories could not be matched to the film (95.9\% did match) and were not included in analyses. The mean emotion rating of the intrusive memories was 2.15 (S.D. $=0.45$ ), suggesting that participants found their intrusive memories negative.

The number of different scene types per person was the variable of interest for the fMRI analysis. The mean number of Intrusive scenes per participant was 3.09
(S.D. =1.46), leaving a mean number of Potential scenes (from the 20 possible scenes) of 16.91 (S.D. =1.46). The number of Control scenes was pre-determined at 16 per participant.

On the recognition memory test at 1 week, in our set, Intrusive picture stills were better recognized than Potential picture stills $(83.04 \%$, S.D. $=13.89 ; 64.07 \%$, S.D. $=14.97$, respectively; $\left.t_{34}=6.76, p<0.001\right)$. For further information, see the online Supplementary material.

In the scanner soon after film viewing, 25 participants reported intrusive memories of the film, totalling 148 intrusive memories (mean frequency $=5.92$, S.D. $=$ 4.08; mean number different intrusive memory scenes $=2.36$, S.D. $=1.37$ ). The nine control participants had a mean number of button presses of 7.89 (s.D. $=2.15$ ).

\section{fMRI results}

\section{Intrusive memory encoding}

Whole-brain analysis comparing Intrusive with Potential (Fig. 2a, top row) and Control scenes (Fig. 2a, middle row) revealed widespread increases in activation, including the putamen, rostral anterior cingulate cortex, insula, thalamus and ventral occipital cortex. Signal change extracted from predefined ROIs showed, as predicted, differences in activation in the MTG and left IFG between Intrusive and Potential scenes but not between Intrusive and Control scenes (Fig. 2b). Comparison of Potential scenes with Control scenes at the whole-brain level (Fig. $2 a$ bottom row) revealed increased activation in the thalamus and ventral occipital cortex. Table 1 shows peak voxel coordinates.

Behavioural results on the recognition memory test highlighted that Intrusive picture stills were better recognized than Potential picture stills. Further comparison of the neuroimaging data was therefore made using only those Intrusive and Potential picture stills correctly recognized as from the film to control for any neural effects that could be explained by this better recognition memory. Results show a similar pattern of activation as to the original Intrusive $v$. Potential analysis at both whole-brain (Fig. 2c) and ROI level (Fig. 2d). Table 1 shows peak voxel coordinates. See also online Supplementary material and Fig. S1.

\section{Intrusive memory involuntary recall}

Whole-brain analyses using the five time bins compared activation during intrusive memory involuntary recall (i.e. involuntary recall in the scanner soon after film viewing) and the control button press (Fig. 3a; peak voxel coordinates in Table 2). Increased activation for the intrusive memory involuntary recall group compared with the control button press group was seen in middle and superior frontal regions between 
(a) Scenes Analysis

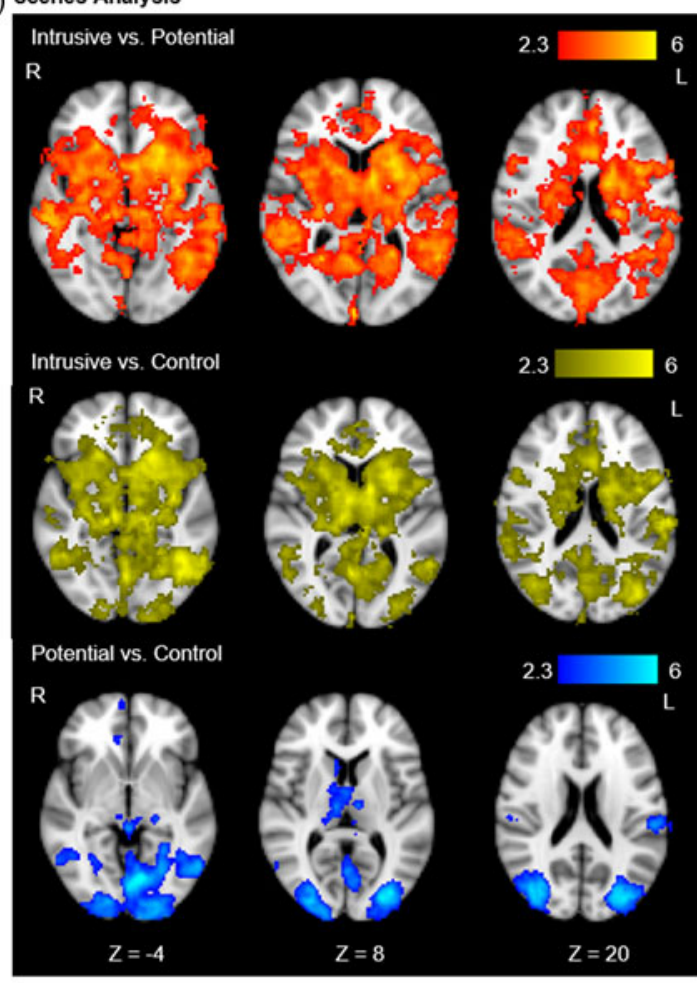

(c) Stills Analysis
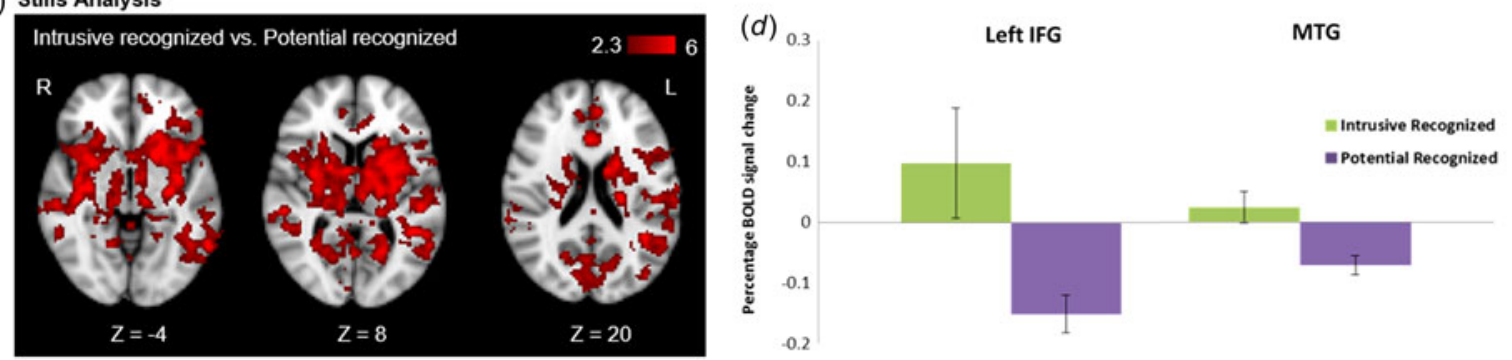

Fig. 2. Neural basis of intrusive memory encoding. (a) Whole-brain analysis of the encoding of Intrusive $v$. Potential $v$. Control scenes, increased blood oxygen level-dependent (BOLD) responses in colour for each contrast. (b) Region-of-interest (ROI) analysis for the left inferior frontal gyrus (IFG) and middle temporal gyrus (MTG) showing the BOLD percentage signal change for Intrusive and Potential scenes relative to Control scenes. (c) Whole-brain analysis of the encoding of Intrusive recognized $v$. Potential recognized, increased BOLD response shown in colour. (d) ROI analysis for the left IFG and MTG showing the BOLD percentage signal change for Intrusive recognized and Potential recognized picture stills. Values are means, with standard deviations represented by vertical bars. R, Right; L, left.

0 and $3 \mathrm{~s}$ and in the left IFG and bilateral operculum between 3 and $6 \mathrm{~s}$. Increased activation for the reverse contrast was seen between 6 and $9 \mathrm{~s}$. No suprathreshold activation was found for either contrast between -3 and 0 , and 9-12 s.

Signal change was extracted from ROIs showing significant activation at the whole-brain level and the precentral gyrus. BOLD signal activation profile plots are shown in Fig. $3 b$. The BOLD signal response in the precentral gyrus followed the same pattern in both groups; peaking during the time bin modelling 3-6 s after the button press. This peak (expected due to the delay in BOLD signal response) suggests successful modelling of changes in activation by the finite impulse response basis functions.

\section{Discussion}

The current study investigated the neural basis of the encoding and involuntary recall of intrusive memories to film footage of traumatic events. We found a widespread pattern of increased activation at the encoding of Intrusive scenes (emotional scenes that were involuntarily recalled) compared with both Potential scenes 
Table 1. Peak voxel coordinates identified in the whole-brain intrusive memory encoding analysis ${ }^{\mathrm{a}}$

\begin{tabular}{|c|c|c|c|c|c|c|}
\hline Analysis & Location & Cluster size & $x$ & $y$ & $z$ & $z$-Statistic \\
\hline \multirow[t]{5}{*}{ Intrusive $>$ potential } & Left putamen & 80991 & -14 & 8 & 2 & 6.08 \\
\hline & Left precuneus & & -22 & -54 & 6 & 5.94 \\
\hline & Left insular & & -36 & 12 & -6 & 5.83 \\
\hline & Left middle temporal gyrus & & -56 & -56 & 10 & 5.82 \\
\hline & Left caudate & & -12 & 14 & 0 & 5.81 \\
\hline \multirow[t]{4}{*}{ Intrusive $>$ control } & Left inferior temporal gyrus & 85347 & -44 & -58 & -6 & 6.37 \\
\hline & Left supramarginal & & -60 & -26 & 30 & 6.27 \\
\hline & Left caudate & & -14 & 10 & 2 & 6.25 \\
\hline & Right thalamus & & 10 & -20 & 10 & 6.18 \\
\hline \multirow[t]{18}{*}{ Potential > control } & Left occipital fusiform gyrus & 30148 & -20 & -70 & -14 & 6.51 \\
\hline & Right supramarginal & & 60 & -22 & 36 & 6.48 \\
\hline & Left lingual gyrus & & -8 & -72 & -6 & 6.45 \\
\hline & Left temporal occipital fusiform cortex & & -26 & -62 & -12 & 6.44 \\
\hline & Right superior parietal & & 22 & -50 & 74 & 6.2 \\
\hline & Right thalamus & 939 & 8 & -16 & 12 & 5.18 \\
\hline & Left thalamus & & -8 & -14 & 12 & 4.14 \\
\hline & Right caudate & & 12 & 0 & 12 & 4.09 \\
\hline & Right superior frontal & 769 & 24 & -6 & 62 & 5.28 \\
\hline & Right middle frontal & & 26 & -2 & 54 & 4.71 \\
\hline & Left superior frontal & 625 & -26 & -4 & 62 & 4.33 \\
\hline & Left precentral & & -22 & -10 & 66 & 3.48 \\
\hline & Right frontal pole & 572 & 14 & 56 & -14 & 4.43 \\
\hline & Right frontal orbital & & 22 & 34 & -12 & 3.81 \\
\hline & Brain stem & 528 & 0 & -34 & -4 & 4.51 \\
\hline & Left thalamus & & -22 & -26 & -4 & 3.44 \\
\hline & Posterior cingulate & & -2 & -38 & 4 & 3.42 \\
\hline & Hippocampus & & -6 & -38 & 6 & 3.25 \\
\hline \multirow{3}{*}{$\begin{array}{l}\text { Intrusive recognized }>\text { potential } \\
\text { recognized }\end{array}$} & Left putamen & 40032 & -20 & 8 & 0 & 4.95 \\
\hline & Left frontal orbital & & -28 & 16 & -18 & 4.84 \\
\hline & Right putamen & & 30 & -16 & 2 & 4.82 \\
\hline
\end{tabular}

${ }^{a}$ Brain regions identified using the Oxford-Harvard cortical and subcortical anatomical atlas.

(emotional scenes that were not involuntarily recalled by that participant but were previously by other participants) and Control scenes (scenes that were never involuntarily recalled). As predicted, the left IFG and MTG showed increased activity between Intrusive $v$. Potential scenes, but not Intrusive $v$. Control scenes. These areas may potentially distinguish whether a scene which is traumatic in content will intrude or not.

The encoding findings provide a strong replication of our previous work (Bourne et al. 2013) in a new sample. In addition to the previous work and importantly, we were able to show that while Intrusive picture stills were better recognized than Potential picture stills, analysis using only recognized picture stills revealed the same pattern of brain activation.

The fMRI involuntary recall findings captured the neural activation at the moment of intrusive memory involuntary recall (that is, experiencing an intrusive memory in the scanner) indicating involvement of the middle and superior frontal cortices, operculum and left IFG. The left IFG was the only region to be involved in both intrusive memory encoding and involuntary recall.

\section{Intrusive memory encoding}

Results provide support for a neural signature at the time of viewing those scenes from footage of traumatic events that later return as an intrusive memory. By comparing activation during Intrusive and Potential picture stills that were recognized at 1 week, we are able to suggest that the differential activation between Intrusive and Potential scenes was not merely due to participants having better recognition memory for Intrusive scenes. That is, we know that all the picture stills used in the analysis were recognized by participants. Neural activation found from comparing intrusive and potential events can therefore not simply be 
(a)
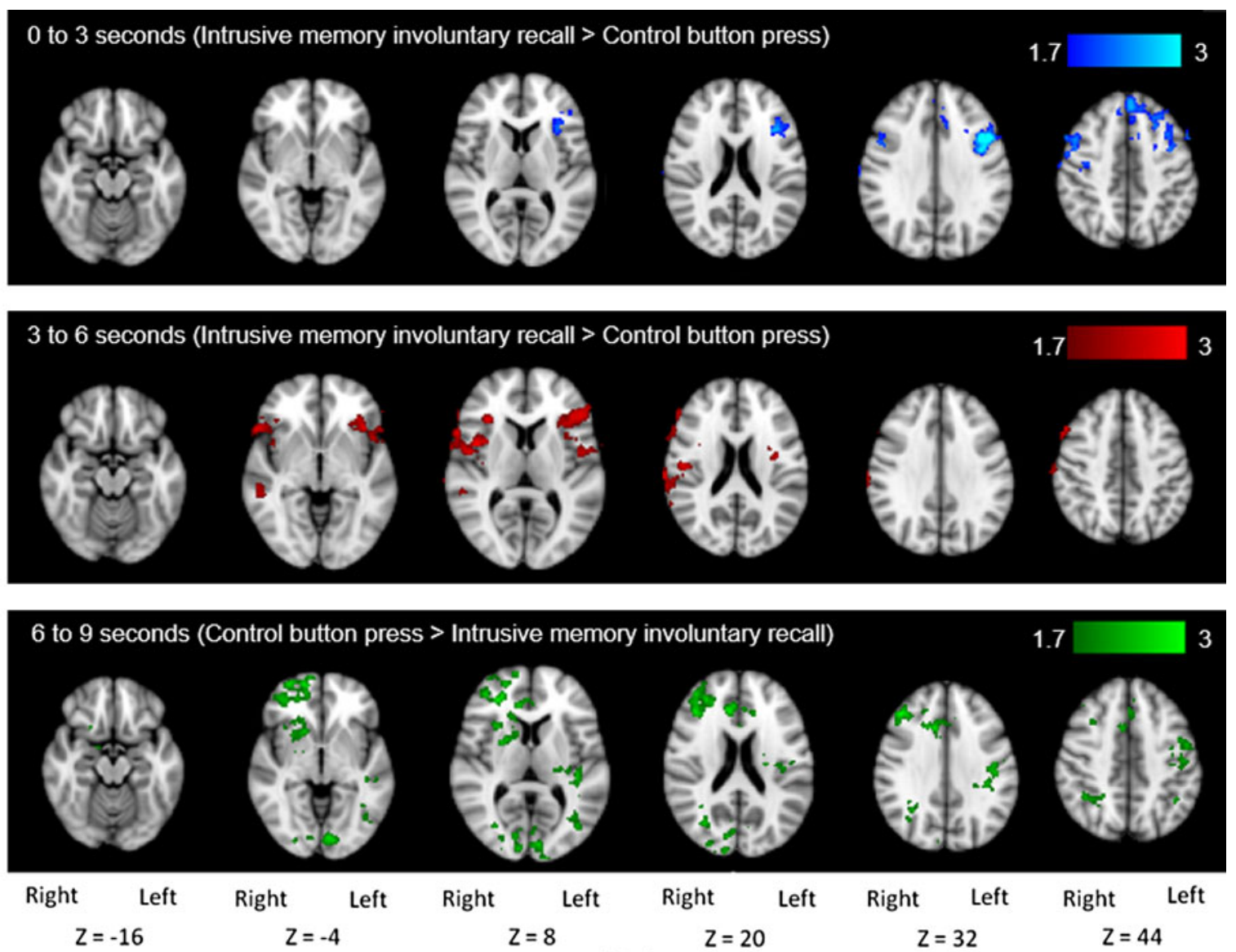

(b)
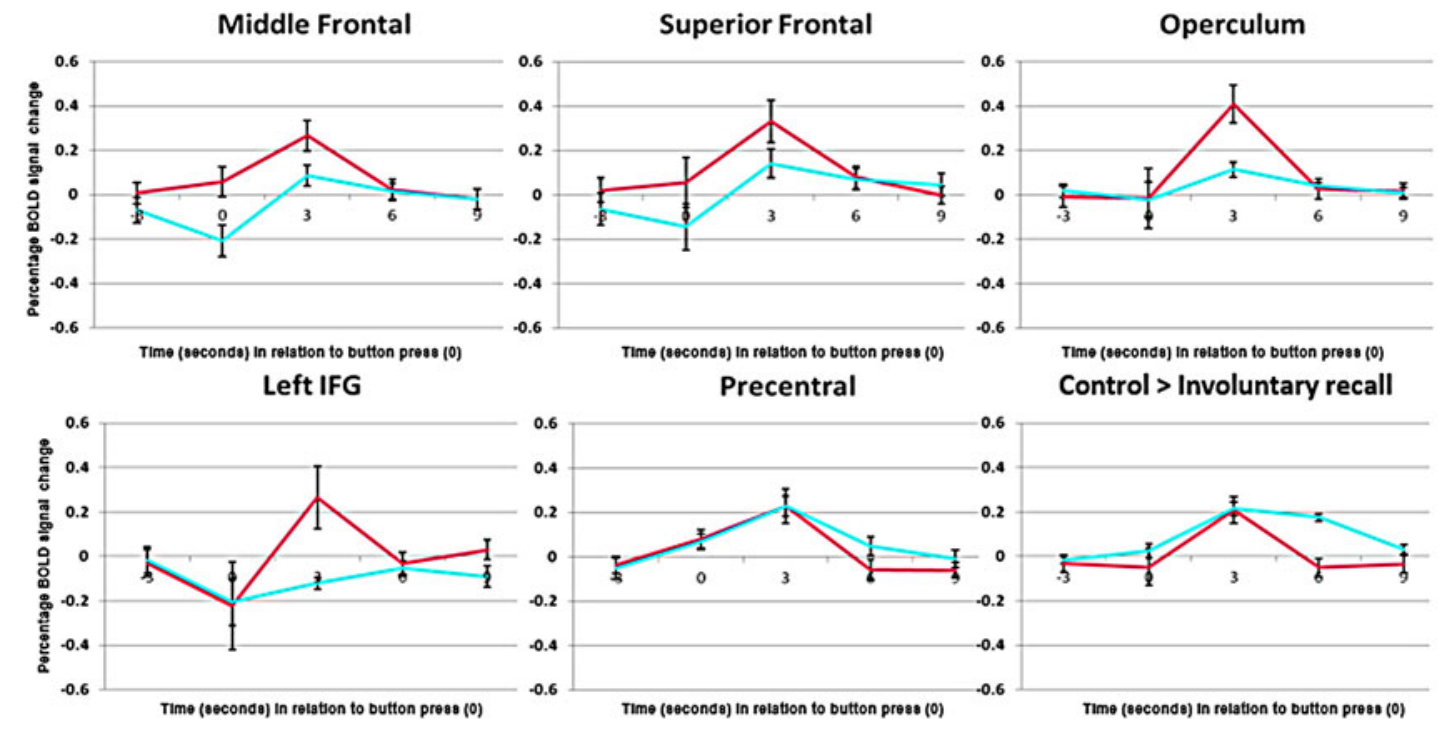

Control > Involuntary recall

Fig. 3. Intrusive memory involuntary recall. (a) Whole-brain analysis showing the increased blood oxygen level-dependent (BOLD) response for intrusive memory involuntary recall $v$. control button press group at the two time bins (0-3 s and 3-6 s in relation to the button press) showing significant differences in activation, and the one time bin (6-9 s) showing increased BOLD response for the control button press group $v$. intrusive memory involuntary recall. $(b)$ Region-of-interest profile plots of the signal change observed across each time bin from -3 to $+12 \mathrm{~s}$ in relation to the button press. Intrusive memory involuntary recall signal change activation is shown in pink, control button press signal change activation in light blue. Values are means, with standard deviations represented by vertical bars. IFG, Inferior frontal gyrus. 
Table 2. Peak voxel coordinates identified in the whole-brain intrusive memory involuntary recall analysis ${ }^{\mathrm{a}}$

\begin{tabular}{|c|c|c|c|c|c|c|}
\hline Analysis & Location & Cluster size & $x$ & $y$ & Z & $z$-Statistic \\
\hline \multirow[t]{4}{*}{ Intrusion > control; $0-3 \mathrm{~s}$} & Left middle frontal gyrus & 4312 & -48 & 14 & 32 & 3.39 \\
\hline & Right postcentral & & 54 & -10 & 54 & 3.12 \\
\hline & Superior frontal & & 0 & 10 & 60 & 3.09 \\
\hline & Right precentral & & 44 & -12 & 68 & 3.06 \\
\hline \multirow[t]{9}{*}{ Intrusion $>$ control; $3-6 \mathrm{~s}$} & Right inferior frontal gyrus & 2997 & 60 & 14 & 2 & 3.4 \\
\hline & Right postcentral & & 58 & -12 & 54 & 3.21 \\
\hline & Right middle frontal & & 54 & 18 & 42 & 3.05 \\
\hline & Right central opercular & & 36 & 6 & 16 & 2.96 \\
\hline & Left frontal operculum & 1205 & -32 & 22 & 10 & 3.12 \\
\hline & Left inferior frontal & & -48 & 30 & 8 & 2.78 \\
\hline & Left central opercular & & -46 & -2 & 14 & 2.74 \\
\hline & Left frontal orbital & & -40 & 22 & -4 & 2.68 \\
\hline & Left inferior frontal & & -52 & 36 & 6 & 2.68 \\
\hline \multirow[t]{8}{*}{ Control $>$ intrusion; $6-9 \mathrm{~s}$} & Right middle frontal gyrus & 8180 & 42 & 34 & 28 & 3.24 \\
\hline & Right frontal pole & & 30 & 42 & 10 & 2.92 \\
\hline & Right postcentral & & 26 & -30 & 64 & 2.87 \\
\hline & Left lingual gyrus & 2324 & -6 & -86 & -6 & 2.71 \\
\hline & Right supracalcarine cortex & & 6 & -78 & 16 & 2.66 \\
\hline & Right superior parietal & & 20 & -56 & 72 & 2.6 \\
\hline & Right lateral occipital & & 32 & -66 & 52 & 2.55 \\
\hline & Right intracalcarine cortex & & 14 & -82 & 12 & 2.53 \\
\hline
\end{tabular}

${ }^{\text {a }}$ Brain regions identified using the Oxford-Harvard cortical and subcortical anatomical atlas.

explained as an indication of better recognition memory performance for intrusive events. Indeed, the similarity in brain regions identified by both contrasts supports the notion that neural signature is associated with the later involuntary recall of that event per se.

Multiple regions were associated with the encoding of Intrusive scenes, and included, but were not limited to, regions associated with threat detection, in particular surprise to threat (e.g. rostral anterior cingulate cortex) (Bishop et al. 2004; Browning \& Harmer, 2012), emotional processing, pain and empathy of pain (e.g. insula, anterior cingulate, thalamus) (Leknes \& Tracey, 2008) and visual processing and mental imagery (e.g. ventral occipital cortex) (Kosslyn et al. 2001). These are regions consistent with intrusive memories being emotional, vivid images of traumatic events and regions that are partially in line with models of PTSD (Rauch et al. 2006; Admon et al. 2013).

What might be the theory underlying our neural activation results in the creation of an intrusive memory in comparison with a traumatic moment that does not later return involuntarily? While our experimental design restricts conclusions, we speculate on the following as a starting point for future theoretical development. Neurocircuitry models of PTSD draw on animal fearconditioning models and implicate emotional regions such as the limbic system (e.g. Rauch et al. 2006). Wegerer et al. (2013) have argued that fear conditioning also underlies intrusive memories, albeit in behavioural studies. While our results do highlight emotion regions, in line with fear-conditioning models, a number of additional regions were also identified in our study (e.g. MTG and IFG). This indicates that additional processing beyond that of fear conditioning may be involved (see Beckers et al. 2013).

Several other literatures also provide theoretical insights. Models of intrusive memories in PTSD treatment stemming from clinical and cognitive psychology implicate emotional regions, and additionally point to heightened activity in sensory/imagery-related regions (suggested to be mediated by the precuneus) alongside decreased activity in memory regions (Brewin et al. 2010; Brewin, 2014). This is proposed to lead to ineffective coupling of emotional and contextual information and thus the later occurrence of intrusive memories. Our results are partially consistent with this model (e. g. occipital areas), supporting the emphasis on mental imagery. Notably, imagery is not mentioned in the above neurocircuitry models of PTSD (Rauch et al. 2006). However, we argue that the emphasis on imagery should not be restricted to PTSD memory recall, but rather is part of a continuum with non-clinical autobiographical recall. Episodic memory involves imagery (Tulving, 2002). Vivid image-based autobiographical memories have been associated with activity in occipital regions and the precuneus (Cabeza \& St. Jacques, 2007), 
and the underlying neural processes associated with mental imagery substantially overlap with those for autobiographical memory (Hassabis \& Maguire, 2007; Schacter \& Addis, 2007). This link between autobiographical memory and intrusive memories is also underscored by autobiographical memory theorists who span clinical and non-clinical literatures (Conway, 2001; Berntsen \& Hall, 2004; Rubin et al. 2008).

Where our results notably differ from previous models of intrusive memories and PTSD is the activity pattern found in the left IFG and MTG. The left IFG and MTG showed increased activity between Intrusive and Potential scenes, but not Intrusive and Control scenes. We suggest that these brain regions may be involved in distinguishing why particular traumatic scenes become an intrusive memory while other traumatic scenes in the same sequence do not. As noted in the introduction, both regions have previously been associated with subsequent memory for deliberate recall (Paller \& Wagner, 2002; Kensinger \& Corkin, 2004). We suggest that enhanced encoding occurs at these 'hotspot' moments which later become intrusive memories, with heightened involvement of these memory-related areas in combination with increases in sensory and emotional processing. In contrast, PTSD models proposed elsewhere suggest 'disrupted' encoding and memory fragmentation (e.g. Brewin, 2014).

\section{Intrusive memory involuntary recall during fMRI}

Our final aim of the study was to model brain activity when participants experienced an intrusive memory in the scanner while undergoing fMRI. Using finite impulse response basis functions to model the BOLD signal change we identified neural activity at the moment of intrusive memory involuntary recall. Initial activity was observed in the middle and superior frontal cortices, followed by activation in the operculum and left IFG. These findings of middle and superior frontal cortex activity are convergent with previous results of involuntary recall for picture stimuli (Hall et al. 2008), extending this previous finding to the involuntary recall of more naturalistic complex film stimuli. In PTSD patients, decreases in activity following treatment in the middle frontal cortex during trauma imagery have also been identified (Lindauer et al. 2008). Additionally, the frontal operculum has been associated with the attentional control of cognitive processes and task selection (Higo et al. 2011) and the left IFG with the selection of competing memory representations (Nelson et al. 2009; Levens \& Phelps, 2010).

\section{The left IFG and intrusive memories}

The left IFG was the only region identified here involved in both intrusive memory encoding and involuntary recall. Interestingly, studies of PTSD patients have indicated neural networks involving the left IFG (James et al. 2013) and the left IFG has shown increases in activity when PTSD patients process traumatic compared with neutral material (Landré et al. 2012). Current neurocircuitry models do not implicate the left IFG in PTSD (Rauch et al. 2006; Admon et al. 2013), though we note that this study involves encoding, which by definition has not been examined in PTSD patients.

What might be the role of the left IFG in intrusive memory encoding and recall, at least for experimental trauma? As previously mentioned, the left IFG has been associated with predicting later subsequent memory recall (Kensinger \& Corkin, 2004). It has also been associated with the selection of information (Moss et al. 2005), competing memory representations (Nelson et al. 2009; Levens \& Phelps, 2010) and evaluations of emotional information (Lee \& Siegle, 2012). A meta-analysis of cognitive control suggests that the left IFG may be involved in the 'flexibility' to switch from one task to another (Niendam et al. 2012). Further, greater putamen-left IFG functional connectivity activity has been associated with unwanted thoughts in healthy participants (Kühn et al. 2014). We do note, however, that these associations, while interesting, are made with reverse inference and thus should be done so with caution (Poldrack, 2006).

From our current results, we tentatively hypothesize that left IFG activation while viewing traumatic material (during encoding) may 'flag' the event that will subsequently return as an intrusive memory, comprising an analogue trauma 'hotspot' (Grey \& Holmes, 2008). During intrusive memory involuntary recall, left IFG activation may represent the orientation of attention towards the 'flagged' memory, contributing to the overriding of other psychological functioning and capture of attention.

Overall, our view is that trauma intrusions are not simply bits of 'fragmented or incoherent' memory, rather that intrusions comprise highly selective hotspots meaningful to that individual (Ehlers et al. 2002; Grey \& Holmes, 2008; Krans et al. 2009). Further we see intrusive memory in PTSD on a continuum with other emotional intrusive memories, and on a continuum between clinical and non-clinical populations (see also Kvavilashvili, 2014).

\section{Limitations}

The number of events modelled in the current experiment is low compared with more traditional fMRI designs, though similar to the number of different intrusive memories seen in PTSD patients - a mean of 3.74 (Grey \& Holmes, 2008). This may be inevitable 
in paradigms attempting to capture 'rare' clinically relevant symptoms. Conventional ideas may suggest that three events provide insufficient power for a reliable contrast. On the other hand, the statistics used do account for the low number of events, and a small number of events is more likely to cause a type II error (false negative) than a type I error (false positive), because low event frequency increases noise, making it difficult to find meaningful results (see also empirical demonstration in Bourne et al. 2013; online Supplementary material).

Due to the nature of the traumatic content, scene length varied between 5 and $37 \mathrm{~s}$. This is greater variance than is typically seen in fMRI study designs. Given the slowness of the haemodynamic response (between 5 and $7 \mathrm{~s}$ ), this adds further noise to the data. Further, the total time modelled as specific scenes of interest is relatively low (indeed, the total scan time of the whole film is under $25 \mathrm{~min}$ ). Conventional wisdom suggests scanning for as long as possible and collecting the most data over events of interest as possible, resulting in longer scan times and greater amounts of data (see, for example, Henson, 2007).

However, if our results were detrimentally unreliable due to low event frequency, variance in scene length, limited scanning time or other factors typically enhanced to optimize the fMRI design, we would expect to have been unable to replicate our previous findings. The results presented here on the other hand show a near-identical pattern of activation as our previous results. Further, using multivariate pattern analysis techniques reported elsewhere we have been able to predict intrusive memories solely from the brain activity during encoding of film footage with traumatic content (Clark et al. 2014). Overall, this suggests that these fMRI results underlying intrusive memory encoding, while not ideal in all respects, are reliable in terms of replicability.

The prospective design and use of a scanner at encoding rely upon an analogue of trauma, and this is not the same as experiencing real trauma. However, repeated exposure to media film images of traumatic events have been associated with higher scores on the PTSD Checklist - Civilian Version (a measure of PTSD symptoms) (Silver et al. 2013) and higher acute stress symptoms (Holman et al. 2014). The inclusion of trauma exposure through electronic media, television and movies in the line of work in the new DSM-5 (American Psychiatric Association, 2013) also prompts the need for greater understanding of these forms of exposure.

Our intrusive memory involuntary recall task in the scanner has only been tested here at a time soon after the analogue trauma. Recent evidence suggests that immediate $(1 \mathrm{~h})$ and delayed (1 week) intrusive memories may result from different types of retrieval mechanisms (Staugaard \& Berntsen, 2014). Immediate intrusive memories may relate more to salient aspects of the memorability at encoding (e.g. vividness, emotionality, recency), whereas delayed intrusive memories may reflect the influence of retrieval cues in the environment that elicit such involuntary recall. Our current results are on immediate intrusive memories. Future research should test a larger time interval by returning participants to the scanner at 1 week.

This study aimed to provide the first capture of the neural processes involved at the moment of intrusive memory involuntary recall. However, our intrusive memory involuntary recall analysis presents only the first steps in what will need to be a longer line of enquiry. Our control condition (button press alone) was used to subtract brain activity associated with the button press itself in the absence of an intrusion (for related methodology to capture neural activity associated with the occurrence of a hallucination in schizophrenia via balloon press, see Diederen et al. 2010; Hoffman et al. 2011). Future investigations should develop improved and appropriately powered control conditions to develop methods to capture intrusiveness, as this is key to many psychiatric phenomena. Additionally, further studies which specifically contrast voluntarily and involuntary recall, in particular following movie stimuli, are clearly required.

Finally, it is not possible to ascertain whether the intrusions are 'truly' spontaneous, or merely reported as spontaneous. Interestingly, this issue applies equally to the clinical form of this experimental analogue, since patients with PTSD are asked to report or monitor their spontaneously occurring intrusive memories during assessment/treatment. Future studies might seek to examine this issue further.

\section{Conclusions}

Our analyses suggest that whilst experiencing trauma the brain behaves differently during moments that later become intrusive memories, consistent with clinical suggestions that the peritraumatic phase is important in predicting PTSD (Ozer et al. 2003; American Psychiatric Association, 2013). Whereas a strikingly widespread pattern of activation was involved at encoding, the left IFG was the only region involved in both the encoding and involuntary recall of intrusive memories. What are the clinical implications? Tentatively we suggest that if left IFG activation can be modulated during the encoding of trauma memory and its consolidation (Walker et al. 2003), then we may be able to modulate intrusive memory occurrence by reducing left IFG activation. Further, due to the association between the left IFG and language processing (Vigneau 
et al. 2006), results may provide a clue as to why certain talking-based interventions (e.g. critical incident stress debriefing) soon after trauma have been found to be detrimental (Roberts et al. 2009). Talking-based interventions increasing left IFG activity may serve to increase intrusive memory (re)encoding at this early time point. We note that at later time points (e.g. 1 month and later) trauma-focused cognitive-behavioural therapy is effective (e.g. Bisson et al. 2013).

In summary, after witnessing a traumatic event, it is only certain moments from the trauma that reappear as intrusive memories. Why it is that some moments rather than others become intrusive memories has long been a puzzle. We suggest that alterations in brain activation at the time of viewing trauma determine which moments will later become intrusive memories. In particular, activity in the left IFG seems to be key for both the encoding and the involuntary recall of intrusive memories. Further, we speculate that rather than disrupted encoding resulting in memory fragments and intrusive memories, a theoretical alternative is that intrusive memories result from better encoded memories at specific points in time.

\section{Supplementary material}

For supplementary material accompanying this paper visit http://dx.doi.org/10.1017/S0033291715002007

\section{Acknowledgements}

This work was supported by the UK Medical Research Council (MRC) (I.A.C., MRC Centenary Early Career Award; E.A.H., MRC Intramural Programme MCA060-5PR50), the Wellcome Trust (E.A.H., Wellcome Trust Clinical Fellowship WT088217; M.W.W.), the MRC/Engineering and Physical Sciences Research Council UK MEG Partnership (M.W.W.) and the National Institute for Health Research (NIHR) Oxford Biomedical Research Programme (E.A.H., M.W.W., C.E.M.). The views expressed are those of the authors and not necessarily those of the National Health Service, the NIHR or the Department of Health. Funding to pay the Open Access publication charges for this article was provided by the UK Medical Research Council.

\section{Declaration of Interest}

None.

\section{References}

Admon R, Milad MR, Hendler T (2013). A causal model of post-traumatic stress disorder: disentangling predisposed from acquired neural abnormalities. Trends in Cognitive Sciences 17, 337-347.

American Psychiatric Association (2013). Diagnostic and Statistical Manual of Mental Disorders, 5th edn. American Psychiatric Association: Washington, DC.

Beckers T, Krypotos A-M, Boddez Y, Effting M, Kindt M (2013). What's wrong with fear conditioning? Biological Psychology 92, 90-96.

Beckmann CF, Jenkinson M, Smith SM (2003). General multi-level linear modelling for group analysis in fMRI. NeuroImage 20, 1052-1063.

Berntsen D, Hall NM (2004). The episodic nature of involuntary autobiographical memories. Memory and Cognition 32, 789-803.

Bishop S, Duncan J, Brett M, Lawrence AD (2004). Prefrontal cortical function and anxiety: controlling attention to threat-related stimuli. Nature Neuroscience 7, 184-188.

Bisson JI, Roberts NP, Andrew M, Cooper R, Lewis C (2013). Psychological therapies for chronic post-traumatic stress disorder (PTSD) in adults. Cochrane Database of Systematic Reviews, Issue 12. Art. No.: CD003388. DOI: 10.1002/ 14651858.CD003388.pub4.

Bourne C, Mackay CE, Holmes EA (2013). The neural basis of flashback formation: the impact of viewing trauma. Psychological Medicine 43, 1521-1533.

Breslau N, Kessler RC, Chilcoat HD, Schultz LR, Davis GC, Andreski A (1998). Trauma and posttraumatic stress disorder in the community. Archives of General Psychiatry 55, 626-632.

Brewin CR (2013). "I Wouldn't Start From Here" - an alternative perspective on PTSD from the ICD-11: comment on Friedman (2013). Journal of Traumatic Stress 26, 557-559.

Brewin CR (2014). Episodic memory, perceptual memory, and their interaction: foundations for a theory of posttraumatic stress disorder. Psychological Bulletin 140, 69-97.

Brewin CR, Gregory JD, Lipton M, Burgess N (2010). Intrusive images in psychological disorders: characteristics, neural mechanisms, and treatment implications.

Psychological Review 117, 210-232.

Browning M, Harmer CJ (2012). Expectancy and surprise predict neural and behavioral measures of attention to threatening stimuli. NeuroImage 59, 1942-1948.

Bryant RA, McGrath C, Felmingham KL (2013). The roles of noradrenergic and glucocorticoid activation in the development of intrusive memories. PLOS ONE 8, e62675.

Buxton RB, Uludağ K, Dubowitz DJ, Liu TT (2004). Modeling the hemodynamic response to brain activation. NeuroImage 23 (Suppl. 1), S220-S233.

Cabeza R, St. Jacques P (2007). Functional neuroimaging of autobiographical memory. Trends in Cognitive Sciences 11, 49-57.

Clark IA, Niehaus KE, Duff EP, Di Simplicio MC, Clifford GD, Smith SM, Mackay CE, Woolrich MW, Holmes EA (2014). First steps in using machine learning on fMRI data to predict intrusive memories of traumatic film footage. Behaviour Research and Therapy 62, 37-46.

Conway MA (2001). Sensory-perceptual episodic memory and its context: autobiographical memory. Philosophical Transactions of the Royal Society of London Series B - Biological Sciences 356, 1375-1384. 
Diederen KM, Neggers SF, Daalman K, Blom JD, Goekoop R, Kahn RS, Sommer IE (2010). Deactivation of the parahippocampal gyrus preceding auditory hallucinations in schizophrenia. American Journal of Psychiatry 167, 427-435.

Ehlers A, Clark DM (2000). A cognitive model of posttraumatic stress disorder. Behaviour Research and Therapy 38, 319-345.

Ehlers A, Hackmann A, Steil R, Clohessy S, Wenninger K, Winter $\mathbf{H}$ (2002). The nature of intrusive memories after trauma: the warning signal hypothesis. Behaviour Research and Therapy 40, 995-1002.

Forman SD, Cohen JD, Fitzgerald M, Eddy WF, Mintun MA, Noll DC (1995). Improved assessment of significant activation in functional magnetic resonance imaging (fMRI): use of a cluster-size threshold. Magnetic Resonance in Medicine 33, 636-647.

Grey N, Holmes EA (2008). "Hotspots" in trauma memories in the treatment of post-traumatic stress disorder: a replication. Memory 16, 788-796.

Hall NM, Gjedde A, Kupers R (2008). Neural mechanisms of voluntary and involuntary recall: a PET study. Behavioural Brain Research 186, 261-272.

Hassabis D, Maguire EA (2007). Deconstructing episodic memory with construction. Trends in Cognitive Sciences 11, 299-306.

Henson RN (2007). Efficient experimental design for fMRI. In Statistical Parametric Mapping. The Analysis of Functional Brain Images (ed. K. J. Friston, J. Ashburner, S. Kiebel, T. E. Nichols and W. D. Penny), pp. 193-210. Academic Press: London.

Higo T, Mars RB, Boorman ED, Buch ER, Rushworth MFS (2011). Distributed and causal influence of frontal operculum in task control. Proceedings of the National Academy of Sciences USA 108, 4230-4235.

Hoffman RE, Pittman B, Constable RT, Bhagwagar Z, Hampson M (2011). Time course of regional brain activity accompanying auditory verbal hallucinations in schizophrenia. British Journal of Psychiatry 198, 277-283.

Holman EA, Garfin DR, Silver RC (2014). Media's role in broadcasting acute stress following the Boston marathon bombings. Proceedings of the National Academy of Sciences USA 111, 93-98.

Holmes EA, Bourne C (2008). Inducing and modulating intrusive emotional memories: a review of the trauma film paradigm. Acta Psychologica 127, 553-566.

Holmes EA, Brewin CR, Hennessy RG (2004). Trauma films, information processing, and intrusive memory development. Journal of Experimental Psychology: General 133, 3-22.

Hughes KC, Shin LM (2011). Functional neuroimaging studies of post-traumatic stress disorder. Expert Review of Neurotherapeutics 11, 275-285.

James LM, Engdahl BE, Leuthold AC, Lewis SM, Van Kampen E, Georgopoulos AP (2013). Neural network modulation by trauma as a marker of resilience: differences between veterans with posttraumatic stress disorder and resilient controls. JAMA Psychiatry 70, 410-418.

Kensinger EA, Corkin S (2004). Two routes to emotional memory: distinct neural processes for valence and arousal.
Proceedings of the National Academy of Sciences USA 101, 3310-3315.

Kosslyn SM, Ganis G, Thompson WL (2001). Neural foundations of imagery. Nature Reviews Neuroscience 2, 635-642.

Krans J, Naring G, Becker ES, Holmes EA (2009). Intrusive trauma memory: a review and functional analysis. Applied Cognitive Psychology 23, 1076-1088.

Kühn S, Vanderhasselt M-A, De Raedt R, Gallinat J (2014). The neural basis of unwanted thoughts during resting state. Social Cognitive and Affective Neuroscience 9, 1320-1324.

Kvavilashvili L (2014). Solving the mystery of intrusive flashbacks in posttraumatic stress disorder: comment on Brewin. Psychological Bulletin 140, 98-104.

Landré L, Destrieux C, Andersson F, Barantin L, Quidé $Y$, Tapia G, Jaafari N, Clarys D, Gaillard P, Isingrini M, El-Hage W (2012). Working memory processing of traumatic material in women with posttraumatic stress disorder. Journal of Psychiatry and Neuroscience 37, 87-94.

Lanius RA, Bluhm R, Lanius U, Pain C (2006). A review of neuroimaging studies in PTSD: heterogeneity of response to symptom provocation. Journal of Psychiatric Research 40, 709-729.

Lazarus RS (1964). A laboratory approach to the dynamics of psychological stress. American Psychologist 19, 400-411.

Lee KH, Siegle GJ (2012). Common and distinct brain networks underlying explicit emotional evaluation: a meta-analytic study. Social Cognitive and Affective Neuroscience 7, 521-534.

Leknes S, Tracey I (2008). A common neurobiology for pain and pleasure. Nature Reviews Neuroscience 9, 314-320.

Levens SM, Phelps EA (2010). Insula and orbital frontal cortex activity underlying emotion interference resolution in working memory. Journal of Cognitive Neuroscience 22, 2790-2803.

Liberzon I, Taylor SF, Fig LM, Koeppe RA (1997). Alterations of corticothalamic perfusion ratios during a PTSD flashback. Depression and Anxiety 4, 146-150.

Lindauer RJL, Booij J, Habraken JBA, van Meijel EPM, Uylings HBM, Olff M, Carlier IVE, den Heeten GJ, van Eck-Smit BLF, Gersons BPR (2008). Effects of psychotherapy on regional cerebral blood flow during trauma imagery in patients with post-traumatic stress disorder: a randomized clinical trial. Psychological Medicine 38, 543-554.

Moss HE, Abdallah S, Fletcher P, Bright P, Pilgrim L, Acres K, Tyler LK (2005). Selecting among competing alternatives: selection and retrieval in the left inferior frontal gyrus. Cerebral Cortex 15, 1723-1735.

Nelson JK, Reuter-Lorenz PA, Persson J, Sylvester C-YC, Jonides J (2009). Mapping interference resolution across task domains: a shared control process in left inferior frontal gyrus. Brain Research 1256, 92-100.

Niendam TA, Laird AR, Ray KL, Dean YM, Glahn DC, Carter CS (2012). Meta-analytic evidence for a superordinate cognitive control network subserving diverse executive functions. Cognitive, Affective and Behavioral Neuroscience 12, 241-268.

Osuch EA, Benson B, Geraci M, Podell D, Herscovitch P, McCann UD, Post RM (2001). Regional cerebral blood flow correlated with flashback intensity in patients with posttraumatic stress disorder. Biological Psychiatry 50, 246-253. 
Ozer EJ, Best SR, Lipsey TL, Weiss DS (2003). Predictors of posttraumatic stress disorder and symptoms in adults: a meta-analysis. Psychological Bulletin 129, 52-73.

Paller KA, Wagner AD (2002). Observing the transformation of experience into memory. Trends in Cognitive Science 6, 93-102.

Pietrzak RH, Henry S, Southwick SM, Krystal JH, Neumeister A (2013). Linking in vivo brain serotonin type $1 \mathrm{~B}$ receptor density to phenotypic heterogeneity of posttraumatic stress symptomatology. Molecular Psychiatry 18, 399-401.

Poldrack RA (2006). Can cognitive processes be inferred from neuroimaging data? Trends in Cognitive Sciences 10, 59-63.

Rauch SL, Shin LM, Phelps EA (2006). Neurocircuitry models of posttraumatic stress disorder and extinction: human neuroimaging research - past, present, and future. Biological Psychiatry 60, 376-382.

Rauch SL, Shin LM, Whalen PJ, Pitman RK (1998). Neuroimaging and the neuroanatomy of PTSD. CNS Spectrums 3 (Suppl. 2), 30-41.

Rauch SL, van der Kolk BA, Fisler RE, Alpert NM, Orr SP, Savage CR, Fischman AJ, Jenike MA, Pitman RK (1996). A symptom provocation study of posttraumatic stress disorder using positron emission tomography and script-driven imagery. Archives of General Psychiatry 53, 380-387.

Roberts NP, Kitchiner NJ, Kenardy J, Bisson JI (2009). Multiple session early psychological interventions for the prevention of post-traumatic stress disorder. Cochrane Database of Systematic Reviews, Issue 3. Art. No.: CD006869. DOI: 10.1002/14651858.CD006869.pub2.

Rubin DC, Boals A, Bernsten D (2008). Memory in posttraumatic stress disorder: properties of voluntary and involuntary, traumatic and non-traumatic autobiographical memories in people with and without PTSD symptoms. Journal of Experimental Psychology: General 137, 591-614.

Schacter DL, Addis DR (2007). The cognitive neuroscience of constructive memory: remembering the past and imagining the future. Philosophical Transactions of the Royal Society of Biological Sciences 362, 773-786.

Shin LM, Kosslyn SM, McNally RJ, Alpert NM, Thompson WL, Rauch SL, Macklin ML, Pitman RK (1997). Visual imagery and perception in posttraumatic stress disorder. A positron emission tomographic investigation. Archives of General Psychiatry 54, 233-241.
Shin LM, McNally J, Kosslyn SM, Thompson WL, Rauch SL, Alpert NM, Metzger LJ, Lasko NB, Orr SP, Pitman RK (1999). Regional cerebral blood flow during script-driven imagery in childhood sexual abuse-related PTSD: a PET investigation. American Journal of Psychiatry 156, 575-584.

Shin LM, Orr SP, Carson MA, Rauch SL, Macklin ML, Lasko NB, Peters PM, Metzger LJ, Dougherty DD, Cannistraro PA, Alpert NM, Fischman AJ, Pitman RK (2004). Regional cerebral blood flow in the amygdala and medial prefrontal cortex during traumatic imagery in male and female Vietnam veterans with PTSD. Archives of General Psychiatry 61, 168-176.

Shin LM, Rauch SL, Pitman RK (2006). Amygdala, medial prefrontal cortex, and hippocampal function in PTSD. Annals of the New York Academy of Sciences 1071, 67-79.

Silver RC, Holman EA, Andersen JP, Poulin M, McIntosh DN, Gil-Rivas V (2013). Mental- and physical-health effects of acute exposure to media images of the September 11, 2001, attacks and the Iraq war. Psychological Science 24, 1623-1634.

Staugaard SR, Berntsen D (2014). Involuntary memories of emotional scenes: the effects of cue discriminability and emotion over time. Journal of Experimental Psychology: General 143, 1939-1957.

Tulving E (2002). Episodic memory: from mind to brain. Annual Review of Psychology 53, 1-25.

Vigneau M, Beaucousin V, Hervé PY, Duffau H, Crivello F, Houdé O, Mazoyer B, Tzourio-Mazoyer N (2006).

Meta-analyzing left hemisphere language areas: phonology, semantics, and sentence processing. NeuroImage 30, 1414-1432.

Walker MP, Brakefield T, Hobson JA, Stickgold R (2003). Dissociable stages of human memory consolidation and reconsolidation. Nature 425, 616-620.

Wegerer M, Blechert J, Kerschbaum H, Wilhelm FH (2013). Relationship between fear conditionability and aversive memories: evidence from a novel conditioned-intrusion paradigm. PLOS ONE 8, e79025.

Woolrich MW (2008). Robust group analysis using outlier inference. NeuroImage 41, 286-301.

Woolrich MW, Behrens T, Beckmann CF, Jenkinson M, Smith SM (2004). Multi-level linear modelling for fMRI group analysis using Bayesian inference. NeuroImage 21, 1732-1747. 